\title{
Evolving role of cetuximab in the treatment of colorectal cancer
}

This article was published in the following Dove Press journal:

Cancer Management and Research

22 July 2009

Number of times this article has been viewed

\section{Gunter Schuch \\ Sebastian Kobold \\ Carsten Bokemeyer \\ Department of Oncology, Hematology, and Bone Marrow Transplantation with Section of Pneumology, University Medical Center Hamburg-Eppendorf, Hamburg, Germany}

Correspondence: Gunter Schuch Department of Oncology, Hematology, and Bone Marrow Transplantation with Section of Pneumology, University Medical Center Hamburg-Eppendorf, Martinistr, 52, 20246 Hamburg, Germany Tel +497410 54315

Fax+497410 56784

Email schuch@uke.uni-hamburg.de

\begin{abstract}
In recent years, the monoclonal epidermal growth factor receptor (EGFR)-targeting antibody cetuximab was introduced into systemic therapy of colorectal cancer and gained an established role in the treatment of this disease. Cetuximab was shown to be active as a single agent in chemorefractory metastatic disease as well as in combination with varying chemotherapies. Recently, randomized trials demonstrated the activity of cetuximab combinations in the first-line setting of metastatic colorectal cancer. Interestingly, the activity of cetuximab was restricted to patients with $K R A S$ wildtype tumors, as was seen with panitumumab, another EGFR antibody. While $60 \%-70 \%$ of tumors harbor $K R A S$ wildtype genes, $30 \%-40 \%$ of tumors express oncogenic KRAS with mutations in codons 12 and 13 causing constitutive activation of signaling cascades downstream of EGFR and resistance to EGFR blockade. Since proof of $K R A S$ wildtype status became a prerequisite for cetuximab treatment, KRAS testing is being established throughout the world. Future trials will address the question which part of the $K R A S$ wildtype cohort will benefit from EGFR inhibition and how to identify those patients. Additionally, new strategies for treatment of $K R A S$ mutated tumors are strongly needed. Recent developments and future strategies will be summarized.
\end{abstract}

Keywords: cetuximab, colorectal cancer, KRAS

\section{Initial clinical development in refractory colorectal cancer}

Colorectal cancer is one of the leading tumor types worldwide with about $25 \%$ of patients having metastatic disease at diagnosis. ${ }^{1}$ Additionally, patients with stage II or III disease are at considerable risk to develop recurrence and metastases after curative resection. The median survival time of patients with stage IV disease receiving best supportive care only is limited to around 3-6 months. Treatment with 5FU in combination with folinic acid in metastatic disease prolonged survival up to 12 months. $^{2}$ The introduction of irinotecan and oxaliplatin has significantly improved median survival times of colorectal cancer patients to around 20-24 months when all treatment options are given sequentially. ${ }^{3,4}$

In the last decade new targets have been identified for the treatment of colon cancer and specific drugs have been introduced into patient care. Especially the receptor for epidermal growth factor (EGFR) gained much interest. EGFR is expressed on more than $80 \%$ of colorectal cancer cells and preclinical data demonstrated its central role in tumor-specific functions such as proliferation, invasion, metastasis, and angiogenesis. ${ }^{5-8}$ Cetuximab was initially developed by ImClone Systems (New York, NY, USA) as a specific monoclonal antibody to bind and block EGFR signaling. Cetuximab is an 
immunoglobulin G1 (IgG1)-specific human mouse chimeric antibody that demonstrated activity in preclinical models of colorectal cancer. $^{7-9}$

A phase II trail of cetuximab monotherapy in 57 patients with chemorefractory colorectal cancer demonstrated modest activity with $9 \%$ response rate and a median survival of 6.4 months. ${ }^{10}$ The so-called BOND trial investigated cetuximab either as monotherapy or in combination with irinotecan in patients refractory to irinotecan. Cetuximab monotherapy was associated with a response rate of $10.8 \%$ and a median survival of 6.9 months in these heavily pretreated patients. The combination of cetuximab with irinotecan improved the response rate to $22.9 \%$ and median survival was estimated with 8.6 months. ${ }^{11}$ Based on these data cetuximab was approved for the treatment of irinotecan-refractory colon cancer by the US Food and Drug Administration (FDA) in February 2004 and by the European Medicines Agency (EMEA) in June 2004. Data of the cetuximab-irinotecan combination in refractory patients were confirmed in a single center phase II trial verifying a response rate of $25 \%$ and a median survival of 9.8 months. ${ }^{12}$ Similar results were reported in a multicenter phase II trial from Japan confirming almost identical efficacy data in an Asian population. ${ }^{13}$

\section{Further development as second- and third-line therapy}

A large multinational trial was designed to confirm the results of the BOND trial in a heavily pretreated population with patients progressing on irinotecan-containing regimens. 1147 patients with EGFR-positive colorectal cancer received cetuximab in combination with various irinotecan schedules, either weekly, every two weeks, or every three weeks. Response rates ranged between $17.3 \%$ and $21.4 \%$ depending on the irinotecan schedule. A mean overall survival (OS) time of 9.2 months was reached. ${ }^{14}$ This so-called MABEL trial confirmed the activity of cetuximab added to irinotecan in irinotecan-pretreated patients in a large population in the community setting. Infusion-related reactions of grades 3 and 4 were observed in less than $1 \%$ of patients. Severity of acnelike rash was associated with improved efficacy parameters such as progression-free survival (PFS).

Beside the original schedule of weekly cetuximab several trials attempted to investigate whether a more convenient schedule with infusions every other week might influence pharmacokinetics and efficacy of cetuximab. Similar efficacy results and toxicity data were observed in a schedule of cetuximab in a dose of $500 \mathrm{mg} / \mathrm{m}^{2}$ and irinotecan $\left(180 \mathrm{mg} / \mathrm{m}^{2}\right)$ given every two weeks as compared to previous data with weekly cetuximab. ${ }^{15,16}$ However, the two-weekly applications have not been approved yet.

Further attempts to improve the efficacy of chemotherapy in combination with cetuximab were performed in the second-line setting. Souglakos and colleagues tested the combination of cetuximab with oxaliplatin and capecitabine in patients refractory to first-line oxaliplatin-containing regimen in a phase II trail. The combination of cetuximab with capecitabine and oxaliplatin was safe. The overall response rate in this population was $18.7 \%$. Time to tumor progression however, was short at three months. ${ }^{17}$ In the EPIC trial, patients with oxaliplatin-refractory EGFR-expressing disease were treated with irinotecan either alone or in combination with cetuximab. In this large phase III trail the addition of cetuximab to irinotecan improved the response rate from $4.2 \%$ to $16.4 \%$ and PFS from 2.6 to 4.0 months. The median OS was not different in both groups with 10.0 and 10.7 months. The authors noted that $87 \%$ of patients in the irinotecan arm received cetuximab-containing treatments after disease progression. ${ }^{18}$ This post-study crossover might have washed out survival benefits in the combination group.

\section{Clinical trials in the first-line treatment of metastatic colorectal cancer}

Initial data from first-line treatment with cetuximab were reported from a small cohort of 21 patients with EGFR-positive metastatic colon cancer receiving cetuximab in combination with weekly irinotecan and 5FU/FA with two dose groups of $5 \mathrm{FU}$ (1500 and $2000 \mathrm{mg} / \mathrm{m}^{2}$, respectively). The higher $5 \mathrm{FU}$ dose was associated with diarrhoea and the dose of $1500 \mathrm{mg} / \mathrm{m}^{2}$ was recommended for further trials. In this small patient group the overall response rate (ORR) was 67\% and PFS reached 9.9 months. ${ }^{19}$ Early data from combinations with oxaliplatin were reported from a phase II trial with 43 patients using FOLFOX-4 as chemotherapy backbone. The confirmed ORR was $72 \%$, median PFS was 10.8, and median OS was high with 30 months. Secondary resectability rate was $23 \%$ with resection of liver metastases in curative intention in 10 patients. ${ }^{20} \mathrm{~A}$ small randomized phase II trial investigated the addition of cetuximab to oxaliplatin plus capecitabine in the first-line setting. While an improvement regarding response rate (41\%) and PFS (7.2 months) was observed in the cetuximab arm, the low response rate of $14 \%$ and a PFS of 5.8 months in the control group were of some concern in this trial. ${ }^{21}$ 
Data from a larger population were reported within the OPUS study, a large randomized phase II trial studying FOLFOX-4 with or without cetuximab in 338 untreated patients with positive EGFR staining. Response rate was improved from $36 \%$ to $46 \%$ in the cetuximab group. The difference in response rate was not significant $(\mathrm{p}=0.064)$ for the whole patient population and PFS was identical with 7.2 months in both arms. With increasing data on KRAS mutations in colorectal cancer being associated with lack of response in EGFR-inhibiting therapies, KRAS mutation status was evaluated in 233 patients. In patients with KRAS wildtype tumors the difference in response was highly significant with $37 \%$ vs $61 \%(p=0.011)$ and a lower risk of disease progression was observed. Patients with KRAS mutant tumors did not benefit from the addition of cetuximab. ${ }^{22}$

The CRYSTAL study investigated the combination of cetuximab with FOLFIRI in 1217 untreated patients with colorectal cancer in a randomized fashion. Similar to the
OPUS trial the addition of cetuximab to chemotherapy resulted in an $8 \%$ increase of the ORR $(\mathrm{p}=0.004)$ and PFS was prolonged from 8.0 to 8.9 months $(p=0.0479)$. Interestingly, PFS curves separated late with one-year PFS rates of $23 \%$ and $34 \%$, respectively. When tumor tissue was analyzed for KRAS, mutations were detected in $36 \%$ of patients. In those the addition of cetuximab did not improve response or PFS. In wildtype patients, PFS was prolonged from 8.7 to 9.9 months $(p=0.017) .{ }^{23}$ See Table 1 for an overview of clinical trials.

Based on these data cetuximab has been approved for first-line therapy in metastatic colorectal cancer for patients harboring KRAS wildtype tumors. More details on the role of KRAS will be discussed later.

\section{Cetuximab monotherapy in refractory disease}

Based on a phase II-study and the mono-arm of the BOND trial cetuximab was introduced as monotherapy option in

Table I Selected cetuximab trials. Landmark trials in the development of cetuximab according to clinical treatment lines with main characteristics and results such as progression-free survival (PFS) and response rate (RR). In several trials time to progression (TTP) was used as clinical endpoint (in parentheses). Recent studies analyzed efficacy results for KRAS wildtype patients separately. Data of wildtype cohorts are noted (italic) below results of the entire populations

\begin{tabular}{|c|c|c|c|c|c|c|}
\hline Author & Phase & $\begin{array}{l}\text { Patients } \\
\text { (n) }\end{array}$ & Treatment line & Therapy & PFS (mo) & RR (\%) \\
\hline Saltz et al 2004 & II & 57 & $\geq 2$ nd (irinotecan refr.) & Cet mono & 1.4 & 9 \\
\hline $\begin{array}{l}\text { Cunningham } \\
\text { et al } 2004\end{array}$ & III & 329 & $\geq 2$ nd (irinotecan refr.) & $\begin{array}{l}\text { Cet }+ \text { irinotecan vs } \\
\text { Cet mono }\end{array}$ & 4.1 vs 1.5 & 22.9 vs 10.8 \\
\hline Lenz et al 2006 & II & 346 & $\geq 2$ nd (iri + oxali refr.) & Cet mono & 1.4 & 11.6 \\
\hline Wilke et al 2008 & III & 1147 & $\geq 2$ nd (irinotecan refr.) & $\begin{array}{l}\text { Cet }+ \text { irinotecan } \\
\text { (three schedules) }\end{array}$ & 3.2 & 20.1 \\
\hline $\begin{array}{l}\text { Souglakos et al } \\
2007\end{array}$ & II & 40 & $\geq 2$ nd (previous oxaliplatin) & Cet + CapOx & 2.9 (TTP) & 20 \\
\hline $\begin{array}{l}\text { Sobrero et al } \\
2008\end{array}$ & III & 1298 & $\geq 2$ nd (previous oxaliplatin) & $\begin{array}{l}\text { Cet }+ \text { irinotecan vs } \\
\text { irinotecan }\end{array}$ & 4.0 vs 2.6 & I 6.4 vs 4.2 \\
\hline $\begin{array}{l}\text { Folprecht et al } \\
2006\end{array}$ & II & 21 & First line & $\begin{array}{l}\text { Cet }+ \text { irinotecan/ } \\
\text { 5FU/FA }\end{array}$ & 9.9 (TTP) & 67 \\
\hline $\begin{array}{l}\text { Tabernero et al } \\
2007\end{array}$ & II & 43 & First line & Cet + FOLFOX & 12.3 & 72 \\
\hline $\begin{array}{l}\text { Borner et al } \\
2008\end{array}$ & II & 74 & First line & XELOX \pm Cet & 7.2 vs 5.8 (TTP) & $4 \mid$ vs 14 \\
\hline $\begin{array}{l}\text { Bokemeyer et al } \\
2009\end{array}$ & II & 337 & First line & FOLFOX-4 \pm Cet & 7.2 vs 7.2 (7.9 vs 7.2$)$ & 46 vs 36 (6I vs 37$)$ \\
\hline $\begin{array}{l}\text { Van Cutsem } \\
\text { et al } 2009\end{array}$ & III & 1198 & First line & FOLFIRI \pm Cet & 8.9 vs 8.0 (9.9 vs 8.7 ) & 46 vs 38 (59 vs 43 ) \\
\hline Saltz et al 2007 & II & 83 & $\geq 2$ nd (previous irinotecan) & Cet + beva \pm irinotecan & 7.3 vs 4.9 (TTP) & 37 vs 20 \\
\hline Tol et al 2009 & III & 755 & First line & CapOx/beva \pm Cet & $\begin{array}{l}9.6 \text { vs } 10.7 \\
(10.5 \text { vs } 10.6)\end{array}$ & $\begin{array}{l}52.7 \text { vs } 50 \\
(61.4 \text { vs } 50)\end{array}$ \\
\hline $\begin{array}{l}\text { Jonker et al } \\
2007\end{array}$ & III & 572 & $\geq 2$ nd & Cet vs BSC & HR 0.68 (3.7 vs I.9) & 8 vs 0 ( 13 vs 0$)$ \\
\hline
\end{tabular}


irinotecan-refractory colorectal cancer. ${ }^{10,11}$ In both trials, a mean response of $10 \%$ was observed. Another phase II trial by Lenz and colleagues demonstrated a similar response rate of $11.6 \%$ and a median OS of 6.6 months in a cohort of 346 patients in chemorefractory patients. ${ }^{24}$ Adverse events included hypersensitivity reactions, acne-like rash, asthenia, diarrhea, and others. Skin toxicities started within 1-3 weeks after initiation of cetuximab treatment. In a phase III setting in heavily pretreated patients, cetuximab was superior to best supportive care in KRAS wildtype patients with a median PFS of 14.8 weeks (mutant 7.2) and meaningful difference in OS of 9.5 months vs 4.5 months in KRAS mutants. ${ }^{25}$

Pessino and colleagues addressed the question whether cetuximab could be used as monotherapy in the first-line setting. Interestingly, the response rate of $10 \%$ in cetuximab monotherapy in chemonaïve patients was similar to that observed in pretreated patients. ${ }^{26}$ Time to progression was only two months. Therefore, cetuximab monotherapy can not be considered as effective first-line treatment.

Findings of anti-EGFR monotherapy have been reviewed recently. $^{27}$

\section{Combination of cetuximab with bevacizumab}

A similar design to the BOND trial, but with the addition of bevacizumab, was tested in irinotecan-refractory patients in a small phase II study. The combination of bevacizumab plus cetuximab resulted in a response rate of $20 \%$ and an OS of 11.4 months in this heavily pretreated cohort. The combination of both antibodies plus irinotecan further improved the response with an ORR of $37 \%$ and OS of 14.5 months. ${ }^{28}$ The results of this BOND-2 study formed the basis for the randomized $\mathrm{CAIRO} 2$ trial investigating the addition of cetuximab to a combination of capecitabine, oxaliplatin, and bevacizumab. Although no unexpected toxicities were observed, ${ }^{29}$ efficacy data were surprisingly inferior for the cetuximab-treated patients. In the intention-to-treat (ITT) population PFS was 10.7 months in the control group and 9.4 months in the cetuximab arm. In the subanalysis of the KRAS wildtype population there was no difference in PFS and OS between treatment arms. In KRAS mutant patients PFS was significantly lower in the cetuximab arm with 8.6 months. Interestingly, the control group had a higher PFS (12.5 months) compared to all other groups. ${ }^{30}$

A similar attempt using the EGFR antibody panitumumab was studied in the PACCE trial. In this randomized firstline trial panitumumab was added to either oxaliplatinor irinotecan-based chemotherapy plus bevacizumab.
The combination was associated with inferior outcome as compared to the control arm with PFS times of 10 vs 11.4 months and OS of 19.4 vs 24.5 months for panitumumab and the control arm, respectively. Additionally, toxicities were significantly increased in the panitumumab arm. KRAS analyses resulted in inconsistent data between groups. Oxaliplatin-based chemotherapy plus both antibodies was associated with inferior response compared to the control group in KRAS wildtype patients. ${ }^{31}$

Based on these two negative randomized trials, the idea of EGFR and vascular endothelial growth factor (VEGF) double-targeting with monoclonal antibodies has failed. The underlying mechanism is not understood so far. One explanation could be the downregulation of VEGF production under EGFR inhibition. This may cause activation of alternative proangiogenic factors and lack of efficiency of VEGF blockade.

\section{The biology of KRAS in EGFR-targeted treatment of colorectal cancer}

Since the discovery of the epidermal growth factor (EGF) receptor in the early 1980 's, ${ }^{32}$ growing knowledge about ligands, activation, and signaling helped to understand its biological function. ${ }^{33}$ Upon activation, the transmembrane EGF receptor forms a dimer leading to receptor autophosphorylation through its tyrosine kinase activity. ${ }^{34}$ EGFR activates at least five different signaling pathways: the RAS/RAF/MAPK, the phospholipase C, the PI3K/AKT, the STAT, and the SRC pathways. ${ }^{35}$ Of those, RAS/RAF/ MAPK and PI3K/PTEN/AKT pathways are considered as central effectors of EGFR activation. Each of these finally triggers intracellular signals that may support the malignant phenotype. In recent years, increasing evidence suggested that self-activating or loss of function mutations could occur in nearly all signaling pathways and steps.

Besides alterations of p53, FAP, and other oncogenes activating mutations of KRAS have been described as part of the oncogenic transformation during development of colorectal cancer. ${ }^{36}$ Indeed, KRAS mutations have a prevalence of $30 \%-40 \%$ in larger series of colorectal cancer trials. ${ }^{22,23,37}$ While the prognostic role of mutated KRAS in colorectal cancer remains controversial, ${ }^{38,39}$ initial data from small cohorts had suggested, that in patients treated with cetuximab response was only observed in wildtype tumors. ${ }^{40}$ This finding was confirmed by in vitro experiments showing lack of response to cetuximab in colon cancer cells 
expressing mutant KRAS as compared to wildtype cells. ${ }^{41}$ In a larger series of 89 patients among which $27 \%$ had KRAS mutant tumors, wildtype patients had a response rate of $40 \%$ while none of the patients with mutant tumors responded to cetuximab treatment. ${ }^{42}$ These findings were confirmed by another group analyzing 113 patients treated with cetuximab. Early tumor shrinkage was identified as additional predictive marker. ${ }^{43}$

In a randomized phase III trial comparing EGFR inhibition with panitumumab monotherapy to best supportive care in patients refractory to chemotherapy, the objective response for all patients treated with panitumumab was $10 \% .{ }^{44}$ In wildtype patients treated with panitumumab, the response rate was $17 \%$ compared to $0 \%$ in the mutant group. ${ }^{45}$ Based on these data, panitumumab was approved as single agent only for patients with KRAS wildtype tumors.

Almost identical data have been reported from a randomized phase III trail with cetuximab monotherapy versus best supportive care in chemorefractory patients. In this trial enrolling 572 patients, the response rate was $8 \%$ vs $0 \%$ in the cetuximab vs control groups, respectively. ${ }^{46}$ Post-hoc KRAS analyses of $69 \%$ of tumors detected KRAS mutant status in $42 \%$ of patients. In those, there was no difference in PFS and OS when treatment and control groups were compared. In wildtype patients, median OS significantly improved from 4.8 to 9.5 months when cetuximab therapy was given. ${ }^{25}$

The KRAS analyses from the CRYSTAL and OPUS trials confirmed the importance of KRAS mutation status for EGFR-targeted therapy in the first-line treatment of metastatic colorectal cancer. First-line cetuximab in combination with FOLFOX-4 significantly improved the response rate from $37 \%$ to $61 \%$ in KRAS wildtype tumors when cetuximab was added to chemotherapy. PFS was significantly improved from 7.2 to 7.7 months. ${ }^{22}$ A similar effect was observed in the CRYSTAL study using FOLFIRI as backbone with an increase in RR from $43 \%$ to $59 \%$ in wildtype patients and improvement of PFS from 8.7 to 9.9 months. ${ }^{23}$ In the smaller OPUS trial KRAS mutant patients seemed to do worse under cetuximab treatment with lower response rates (49\% vs 33\%) and PFS (8.6 vs 5.8 months) when compared to chemotherapy only. In the CRYSTAL trial there was no significantly inferior outcome in the mutant group. Whether this finding represents a true effect of inferior outcome caused by EGFR inhibition in KRAS mutant tumors in particular in combination with FOLFOX remains unclear.

Based on the presented data, the EMEA approved cetuximab treatment exclusively for patients with KRAS wildtype metastatic colorectal cancer. ${ }^{47}$ The American Society of
Clinical Oncology published a provisional clinical opinion stating that all patients who are candidates for anti-EGFR therapy should have their tumors tested for KRAS mutation status. Patients with KRAS mutations should not receive antiEGFR antibodies. ${ }^{48}$ This development reflected an exciting step towards personalized therapy in solid tumors.

Appropriate and standardized KRAS mutation detection tests are subjects of practical considerations. ${ }^{49}$ Another important question is whether primary and metastases have identical KRAS mutation status. Santini and colleagues analyzed 38 patients with KRAS mutant tumors and found a high concordance of $96 \%$. Only one patient had a wildtype primary and mutant metastases and three patients had mutant primary tumors and wildtype KRAS in their metastases. ${ }^{50}$ Based on this data there is no need to analyze both primary and metastases.

\section{Biomarkers in cetuximab therapy}

In early trials, proof of positive EGFR staining on the tumor tissue was mandatory in order to treat only patients expressing the appropriate target for cetuximab. Further data suggested, that patients with absence of immunhistological EGFR staining might also respond to cetuximab treatment. ${ }^{51,52}$

A larger translational study analyzing 346 patients found no correlation of EGFR-staining score and treatment response. ${ }^{24}$ Although evidence from randomized trials is not available, EGFR immunohistochemical (IHC) staining is no longer required for cetuximab treatment according to current expert opinion. ${ }^{53}$ The lack of EGFR IHC to predict response may be related to the short presentation of receptors on the surface due to receptor turnover.

Further attempts to evaluate meaningful predictive markers for EGFR-blocking agents in colorectal cancer focused primarily on gene amplifications and polymorphisms of the EGFR gene. Increased gene copy numbers of EGFR as detected by fluorescent in situ hybridization (FISH) have been linked to an increased response rate and prolonged OS in cetuximab-treated patients. ${ }^{54}$ Similar results have reported from a panitumumab cohort. ${ }^{55}$ In patients with rectal cancer receiving neoadjuvant radiochemotherapy plus cetuximab, elevated EGFR gene copy numbers were significantly associated with tumor regression. ${ }^{56}$ In contrary, data suggesting no evident association with EGFR gene copy number or mutations have been reported earlier. ${ }^{24}$ Due to the heterogeneity of data and problems in methodological standardization FISH analysis has not entered clinical routine, so far.

Several polymorphisms in the EGFR gene have been identified. Of those, EGFR intron-1 S/S, EGFR $497 \mathrm{G}>\mathrm{A}$ 
and EGFR R521K seem to play considerable roles. ${ }^{57-59}$ In a cohort of 110 patients, increased skin toxicities and treatment response was associated with EGFR intron-1 S/S carriers. ${ }^{57}$

Pharmacogenetic analyzes of peripheral blood allows to examine germ-line variants of relevant genes such as EGFR ligands. Variants in the ligand EGF, namely EGF 61A > G seemed to be associated with an improved OS compared to the A/A genotype. ${ }^{57}$ In another trial, analyses of EGF polymorphisms in KRAS wildtype tumors suggested that EGF 61A > G was significantly associated with decreased response rate and OS. ${ }^{60}$ These contradictory findings need to be re-evaluated in larger cohorts and should be linked to levels of EGF in the serum of patients.

Further potential predictive markers have been identified. Increased expression of EGFR ligands epiregulin and amphiregulin was linked to better disease control rates and longer PFS according to gene array studies analyzing tissue from 110 patients. ${ }^{61}$ Indeed, treatment with cetuximab induces up-regulation of epiregulin, amphiregulin as well as transforming growth factor- $\alpha$ (TGF- $\alpha$ ), another EGFR ligand, in preclinical models and patients treated with cetuximab. ${ }^{62}$

Skin toxicity was reported to correlate with efficacy of cetuximab. ${ }^{14}$ While in patients without skin rash no objective response occurred, patients with increasing rash grades had responses ranging from $7.2 \%$ in mild rash up to $20 \%$ in severe skin toxicity. ${ }^{24}$ The EVEREST trial reported data from doseescalation of cetuximab in patients lacking skin toxicities. Patients receiving escalating doses up to $500 \mathrm{mg} / \mathrm{m}^{2}$ experienced responses in 30\% (13\% in controls) and a median PFS of 4.8 months as compared to 3.9 months in controls. ${ }^{63}$ The lack of response in KRAS mutated tumors demonstrate that skin toxicity and KRAS status are independent predictive markers of cetuximab. ${ }^{64}$

\section{Additional targets in the EGFR signaling cascade}

The role of KRAS mutations has been discovered recently and was discussed before. Aside from KRAS further genes of signaling proteins might be affected by oncogenic mutation and associated with resistance to EGFR inhibition.

Principal effector downstream of KRAS is the serinethreonine-kinase BRAF. A mutation of BRAF with replacement of valine in codon 600 by glutamic acid resulting in an enhanced kinase activity independent from upstream signaling has been described. ${ }^{65}$ This V600E allele mutation occurs in approximately $10 \%$ of colorectal cancer patients. It is associated with microsatellite instability and poor survival of colon cancer patients. ${ }^{66,67} \mathrm{BRAF}$ mutations in colorectal cancer cause resistance to EGFR-targeted therapy with shorter PFS and OS compared to nonmutated patients. ${ }^{68}$ Remarkably, treatment with the BRAF-inhibitor sorafenib in in vitro experiments restored sensitivity to cetuximab in colon cancer cells. ${ }^{68}$

PI3K-activating mutations occur in around 13\% of colorectal cancer patients. ${ }^{69}$ The hot spots are located in exon 9 and 20; they correlate with resistance to cetuximab in metastatic colorectal cancer in vivo and in vitro. ${ }^{70,71}$ On the other hand, Prenen and colleagues reported a series of 200 patients with $12 \%$ PI3K mutants without any correlation of PI3K mutations to cetuximab response. ${ }^{72}$ Therefore, further data on this topic are urgently needed.

The PTEN tumor suppressor is a negative regulator of PI3K signaling. Inactivating mutations or promoter methylation cause loss of expression in $20 \%$ of microsatelliteinstable tumors and was reported to occur in up to $10 \%$ of colorectal cancer patients. ${ }^{73}$ Since loss of PTEN function results in uncontrolled $\mathrm{PI} 3 \mathrm{~K}$ activation, patients do not benefit from EGFR blockade with cetuximab. ${ }^{74-76}$ Interestingly, the concordance between primary tumor and metastases is low with $60 \%$. Only PTEN loss on metastases predicted resistance to cetuximab plus chemotherapy in this patient cohort. ${ }^{76}$ In in vitro experiments the effect of a PTEN-activating drug was shown, but it remains unclear if it would be able to induce re-expression of the protein. ${ }^{77}$

AKT is a serine-threonine-kinase known as a main effector of PI3K signaling. The E17K mutation induces an upstream-signal independent constitutive activation of AKT and resistance to cetuximab. ${ }^{35}$ This signaling pathway can be selectively targeted by mTOR inhibitors. ${ }^{78}$ This approach has already been successfully investigated in metastatic melanoma with constitutively activated PI3K. ${ }^{79}$

No mutations have been described in the STAT pathway, nor was STAT associated with resistance to EGFR targeting, ${ }^{35}$ although, only a small number of samples have been screened.

In the SRC/FAK pathway, activation may be caused by upregulation. ${ }^{35} \mathrm{Src}$ mutations have not been confirmed in colorectal cancer. ${ }^{80}$ In vitro, SRC upregulation has been associated with cetuximab resistance in non-small cell lung cancer cell line. ${ }^{81}$ Remarkably, treatment with the SRC-inhibitor dasatinib restored cetuximab sensitivity. ${ }^{81}$ In addition SRC-inhibitors were found to be effective in colorectal cancer in vitro. ${ }^{82}$

Another interesting target is the IGF1 receptor that can dimerize with EGFR and could directly interact with EGFR downstream signaling and bypass cetuximab blockade. ${ }^{83}$ 


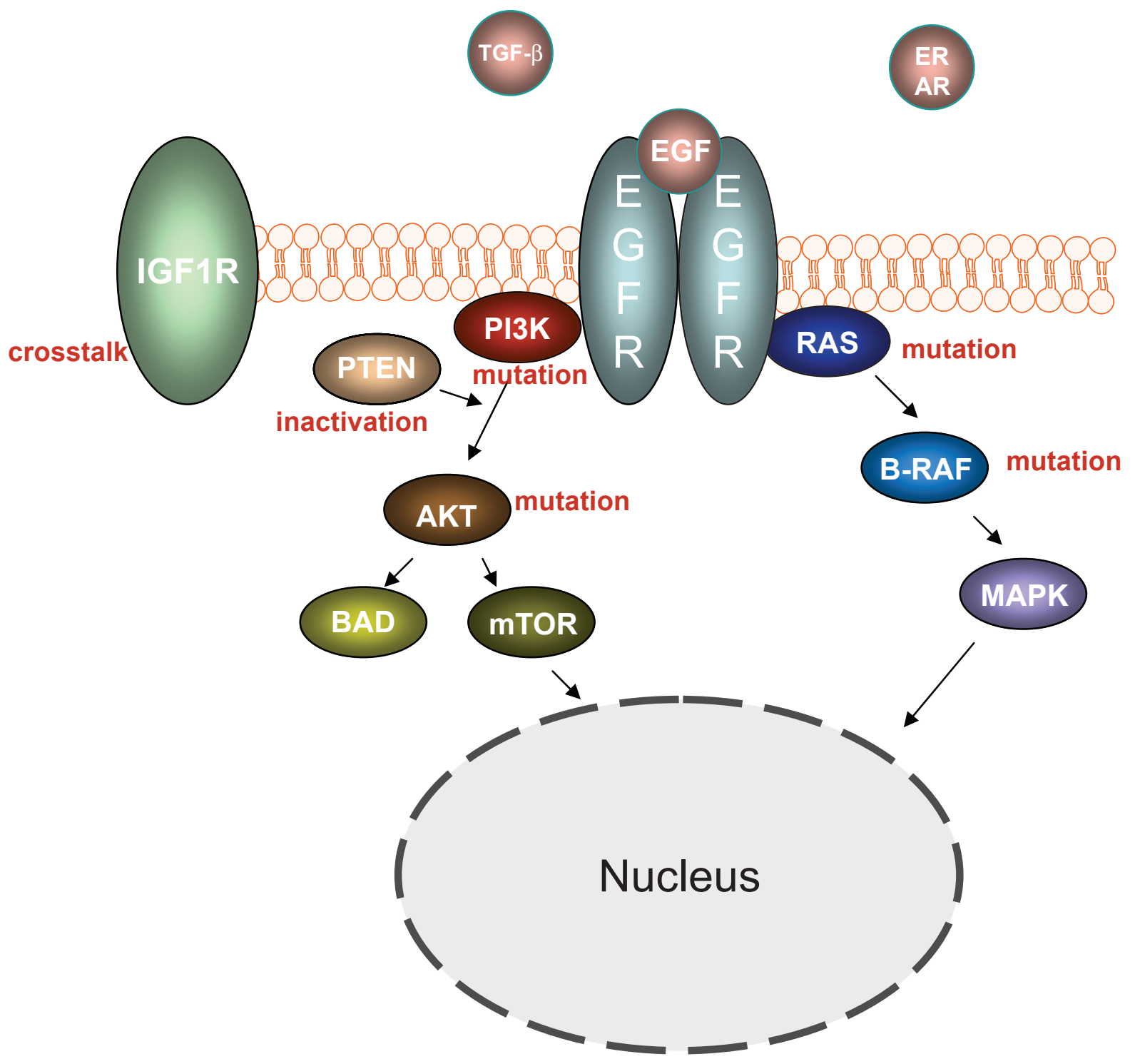

Figure I Simplified EGFR-signaling pathway. Ligands are EGF, TGF- $\beta$, ER, and AR. The two main pathways are the PI3K/AKT/mTOR and RAS/RAF/MAPK cascades. The IGFI receptor can dimerize with EGFR and activate EGFR signaling. Alternatively, downstream crosstalk between signaling cascades may occur. Alterations of downstream effectors known to interfere with EGFR signaling are marked in red.

Abbreviations: AR, amphiregulin; EGF, epidermal growth factor; EGFR, epidermal growth factor receptor; ER, epiregulin; IGFI, insulin-like growth factor-I;TGF- $\beta$, transforming growth factor- $\beta$.

Resistance to EGFR-blocking therapeutics could evolve through alternate mechanisms causing independence of EGFR activation. Beside alterations in the EGFR signal transduction pathway tumors may escape EGFR blockade by increased angiogenesis, activation of alternative tyrosine kinases or receptor mutations (Figure 1). ${ }^{84,85}$

\section{Future directions: immunological biomarkers}

Antibody-dependent cell-mediated cytotoxicity (ADCC) is one of the main modes of therapeutic antibodies such as trastuzumab or rituximab. ${ }^{86,87}$ Polymorphisms in the IgG fragment $\mathrm{C}$ receptor affecting ADCC have been shown to be associated with efficacy of those antibodies. ${ }^{86,87}$ Zhang and colleagues reported two FCGR polymorphisms associated with efficacy of cetuximab monotherapy in 39 patients. ${ }^{88}$ This could be confirmed in another cohort of 69 patients treated with cetuximab plus irinotecan with longer PFS for patients with FCGRIIa-131 H/H and/or FCGRIIIa-158V/V genotypes. The predictive effect of $\mathrm{Fc}$ polymorphisms remained independent of the KRAS status. ${ }^{89}$

Recently, expression of human leukocyte antigen-E (HLA-E) antigen was studied on colorectal cancer tissue. This nonclassical major histocompatibility complex (MHC) 
molecule is overexpressed in human colon cancer and associated with inhibition of natural killer (NK)-mediated cell lyses and might explain the escape to immunological control in this cancer type. Upregulation of HLA-E was also associated with shorter survival of Dukes' C patients. ${ }^{90}$ In vitro, cetuximab-mediated cytotoxicity was hampered in HLA-E overexpressing cells. ${ }^{91}$ While direct antiproliferative effects were seen in cell lines only at high concentrations, ADCC was observed already in low cetuximab concentrations in HLA-E normal-expressors suggesting ADCC as the main effect of cetuximab. The role of HLA-E overexpression needs to be further explored. Probably, it might serve as a new biomarker and future target.

\section{Summary}

EGFR-targeted therapy with cetuximab in colorectal cancer has made significant progress in the recent years. However, the detection of KRAS mutations and their potential impact for treatment guidance opened several new questions. For the future we need to identify patients who will benefit most from EGFR inhibition. This may be available by establishment of a reliable panel of biomarkers that can predict the response to cetuximab. Besides KRAS, this approach involves genetic analyses of ligands and downstream effectors. Additionally, polymorphisms of Fc receptors and HLA-E expression in tumor tissue should be analyzed and correlated to clinical outcome. Based on these evolving data new targets may be identified and could lead to new combinations of targeted drugs. Translational research in medical oncology remains a major challenge in the next years in order to provide the background for personalized treatment of cancer patients.

\section{Disclosure}

The authors report no conflicts of interest in this work.

\section{References}

1. Meyerhardt JA, Mayer RJ. Systemic therapy for colorectal cancer. N Engl J Med. 2005;352:476-487.

2. van Halteren HK, Wagener DJ, Vreugdenhil G, Punt CJ. Advanced colorectal cancer, refractory to infusional fluorouracil treatment: efficacy of second line fluorouracil in combination with a different biochemical modulation. Anticancer Res. 1997;17(4A):2715-2719.

3. Douillard JY, Sobrero A, Carnaghi C, et al. Metastatic colorectal cancer: integrating irinotecan into combination and sequential chemotherapy. Ann Oncol. 2003;14(Suppl 2):ii7-ii12.

4. Tournigand C, Andre T, Achille E, et al. FOLFIRI followed by FOLFOX6 or the reverse sequence in advanced colorectal cancer: a randomized GERCOR study. J Clin Oncol. 2004;22:229-237.

5. Klapper LN, Kirschbaum MH, Sela M, Yarden Y. Biochemical and clinical implications of the ErbB/HER signaling network of growth factor receptors. Adv Cancer Res. 2000;77:25-79.
6. Sato JD, Kawamoto T, Le AD, Mendelsohn J, Polikoff J, Sato GH. Biological effects in vitro of monoclonal antibodies to human epidermal growth factor receptors. Mol Biol Med. 1983;1(5):511-529.

7. Wu X, Fan Z, Masui H, Rosen N, Mendelsohn J. Apoptosis induced by an anti-epidermal growth factor receptor monoclonal antibody in a human colorectal carcinoma cell line and its delay by insulin. J Clin Invest. 1995;95(4):1897-1905.

8. Prewett M, Hooper A, Bassi R, et al. Enhanced antitumor activity of anti-epidermal growth factor receptor monoclonal antibody cetuximab (IMC-C225) in combination with irinotecan (CPT-11), 5-FU, and leucovorin against human colorectal carcinoma xenografts. Eur J Cancer. 2002;38(Suppl 7):150s.

9. Ciardiello F, Bianco R, Damiano V, et al. Antitumor activity of sequential treatment with topotecan and anti-epidermal growth factor receptor monoclonal antibody C225. Clin Cancer Res. 1999;5(4):909-916.

10. Saltz LB, Meropol NJ, Loehrer PJ Sr, et al. Phase II trial of cetuximab in patients with refractory colorectal cancer that expresses the epidermal growth factor receptor. J Clin Oncol. 2004;22:1201-1208.

11. Cunningham D, Humblet $Y$, Siena $S$, et al. Cetuximab monotherapy and cetuximab plus irinotecan in irinotecan-refractory metastatic colorectal cancer. N Engl J Med. 2004;351:337-345.

12. Vincenzi B, Santini D, Rabitti C, et al. Cetuximab and irinotecan as third-line therapy in advanced colorectal cancer patients: a single centre phase II trial. Br J Cancer. 2006;94(6):792-797.

13. Tahara M, Shirao K, Boku N, et al. Multicenter Phase II study of cetuximab plus irinotecan in metastatic colorectal carcinoma refractory to irinotecan, oxaliplatin and fluoropyrimidines. Jpn J Clin Oncol. 2008;38(11):762-769.

14. Wilke H, Glynne-Jones R, Thaler J, et al. Cetuximab plus irinotecan in heavily pretreated metastatic colorectal cancer progressing on irinotecan: MABEL Study. J Clin Oncol. 2008;26(33):5335-5343.

15. Martín-Martorell P, Roselló S, Rodríguez-Braun E, Chirivella I, Bosch A, Cervantes A. Biweekly cetuximab and irinotecan in advanced colorectal cancer patients progressing after at least one previous line of chemotherapy: results of a phase II single institution trial. Br J Cancer. 2008;99(3):455-458.

16. Tabernero J, Pfeiffer P, Cervantes A. Administration of cetuximab every 2 weeks in the treatment of metastatic colorectal cancer: an effective, more convenient alternative to weekly administration? Oncologist. 2008;13(2):113-119.

17. Souglakos J, Kalykaki A, Vamvakas L, et al. Phase II trial of capecitabine and oxaliplatin (CAPOX) plus cetuximab in patients with metastatic colorectal cancer who progressed after oxaliplatin-based chemotherapy. Ann Oncol. 2007;18(2):305-310.

18. Sobrero AF, Maurel J, Fehrenbacher L, et al. EPIC: phase III trial of cetuximab plus irinotecan after fluoropyrimidine and oxaliplatin failure in patients with metastatic colorectal cancer. J Clin Oncol. 2008;26(14):2311-2319.

19. Folprecht G, Lutz MP, Schöffski P, et al. Cetuximab and irinotecan/5-fluorouracil/folinic acid is a safe combination for the first-line treatment of patients with epidermal growth factor receptor expressing metastatic colorectal carcinoma. Ann Oncol. 2006;17(3):450-456.

20. Tabernero J, Van Cutsem E, Díaz-Rubio E, et al. Phase II trial of cetuximab in combination with fluorouracil, leucovorin, and oxaliplatin in the first-line treatment of metastatic colorectal cancer. J Clin Oncol. 2007;25(33):5225-5232.

21. Borner M, Koeberle D, Von Moos R, et al. Adding cetuximab to capecitabine plus oxaliplatin (XELOX) in first-line treatment of metastatic colorectal cancer: a randomized phase II trial of the Swiss Group for Clinical Cancer Research SAKK. Ann Oncol. 2008;19(7):1288-1292.

22. Bokemeyer C, Bondarenko I, Makhson A, et al. Fluorouracil, leucovorin, and oxaliplatin with and without cetuximab in the first-line treatment of metastatic colorectal cancer. J Clin Oncol. 2009;27(5):663-671.

23. Van Cutsem E, Köhne CH, Hitre E, et al. Cetuximab and chemotherapy as initial treatment for metastatic colorectal cancer. $N$ Engl J Med. 2009;360(14):1408-1417. 
24. Lenz HJ, Van Cutsem E, Khambata-Ford S, et al. Multicenter phase II and translational study of cetuximab in metastatic colorectal carcinoma refractory to irinotecan, oxaliplatin, and fluoropyrimidines. J Clin Oncol. 2006;24(30):4914-4921.

25. Karapetis CS, Khambata-Ford S, Jonker DJ, et al. K-ras mutations and benefit from cetuximab in advanced colorectal cancer. $N$ Engl J Med. 2008;359(17):1757-1765.

26. Pessino A, Artale S, Sciallero S, et al. First-line single-agent cetuximab in patients with advanced colorectal cancer. Ann Oncol. 2008;19(4):711-716.

27. Peeters M, Price T, Van Laethem JL. Anti-epidermal growth factor receptor monotherapy in the treatment of metastatic colorectal cancer: where are we today? Oncologist. 2009;14(1):29-39.

28. Saltz LB, Lenz HJ, Kindler HL, et al. Randomized phase II trial of cetuximab, bevacizumab, and irinotecan compared with cetuximab and bevacizumab alone in irinotecan-refractory colorectal cancer: the BOND-2 study. J Clin Oncol. 2007;25(29):4557-4561.

29. Tol J, Koopman M, Rodenburg CJ, et al. A randomised phase III study on capecitabine, oxaliplatin and bevacizumab with or without cetuximab in first-line advanced colorectal cancer, the CAIRO2 study of the Dutch Colorectal Cancer Group (DCCG). An interim analysis of toxicity. Ann Oncol. 2008;19(4):734-738.

30. Tol J, Koopman M, Cats A, et al. Chemotherapy, bevacizumab, and cetuximab in metastatic colorectal cancer. $N$ Engl J Med 2009;360(6):563-572.

31. Hecht JR, Mitchell E, Chidiac T, et al. A randomized phase IIIB trial of chemotherapy, bevacizumab, and panitumumab compared with chemotherapy and bevacizumab alone for metastatic colorectal cancer. J Clin Oncol. 2009;27(5):672-680.

32. Linsley PS, Blifeld C, Wrann M, Fox CF. Direct linkage of epidermal growth factor to its receptor. Nature. 1979;278:745-748.

33. Shin MS, Shinghirunnusorn P, Sugishima Y, et al. Cross interference with TNF-alpha-induced TAK1 activation via EGFR-mediated p38 phosphorylation of TAK1-binding protein 1. Biochim Biophys Acta 2009;1793(7):1156-1164.

34. Ciardiello F, Tortora G. EGFR antagonists in cancer treatment. N Engl J Med. 2008;358:1160-1174.

35. Laurent-Puig P, Lievre A, Blons H. Mutations and response to epidermal growth factor receptor inhibitors. Clin Cancer Res. 2009;15: 1133-1139.

36. Vogelstein B, Fearon ER, Hamilton SR, et al. Genetic alterations during colorectal-tumor development. $N$ Engl J Med. 1988;319(9): 525-532.

37. Andreyev HJ, Norman AR, Cunningham D, Oates JR, Clarke PA. Kirsten ras mutations in patients with colorectal cancer: the multicenter "RASCAL" study. J Natl Cancer Inst. 1998;90(9):675-684.

38. Andreyev HJ, Norman AR, Cunningham D, et al. Kirsten ras mutations in patients with colorectal cancer: the 'RASCAL II' study. Br J Cancer. 2001;85(5):692-696.

39. Bouzourene H, Gervaz P, Cerottini JP, et al. p53 and Ki-ras as prognostic factors for Dukes's stage B colorectal cancer. Eur J Cancer. 2000;36(8):1008-1015.

40. Lièvre A, Bachet JB, Le Corre D, et al. KRAS mutation status is predictive of response to cetuximab therapy in colorectal cancer. Cancer Res. 2006;66(8):3992-3995.

41. Benvenuti S, Sartore-Bianchi A, Di Nicolantonio F, et al. Oncogenic activation of the RAS/RAF signaling pathway impairs the response of metastatic colorectal cancers to anti-epidermal growth factor receptor antibody therapies. Cancer Res. 2007;67(6): 2643-2648.

42. Lièvre A, Bachet JB, Boige $\mathrm{V}$, et al. KRAS mutations as an independent prognostic factor in patients with advanced colorectal cancer treated with cetuximab. J Clin Oncol. 2008;26(3):374-379.

43. De Roock W, Piessevaux H, De Schutter J, et al. KRAS wild-type state predicts survival and is associated to early radiological response in metastatic colorectal cancer treated with cetuximab. Ann Oncol. 2008;19(3):508-515.
44. Van Cutsem E, Peeters M, Siena S, et al. Open-label phase III trial of panitumumab plus best supportive care compared with best supportive care alone in patients with chemotherapy-refractory metastatic colorectal cancer. J Clin Oncol. 2007;25(13):1658-1664.

45. Amado RG, Wolf M, Peeters M, et al. Wild-type KRAS is required for panitumumab efficacy in patients with metastatic colorectal cancer. J Clin Oncol. 2008;26(10):1626-1634.

46. Jonker DJ, O'Callaghan CJ, Karapetis CS, et al. Cetuximab for the treatment of colorectal cancer. N Engl J Med. 2007;357(20):2040-2048.

47. European Medicines Agency (EMEA). European public assessment report: Erbitux-H-558-II-20, 24.11.2008. Accessed on Jun 10, 2009. Available from: http://www.emea.europa.eu/humandocs/Humans/ EPAR/erbitux/erbituxM2.htm.

48. Allegra CJ, Jessup JM, Somerfield MR, et al. American Society of Clinical Oncology provisional clinical opinion: testing for KRAS gene mutations in patients with metastatic colorectal carcinoma to predict response to anti-epidermal growth factor receptor monoclonal antibody therapy. J Clin Oncol. 2009;27(12):2091-2096.

49. Jimeno A, Messersmith WA, Hirsch FR, Franklin WA, Eckhardt SG. KRAS Mutations and Susceptibility to Cetuximab and Panitumumab in Colorectal Cancer. Cancer J. 2009;15(2):110-113.

50. Santini D, Loupakis F, Vincenzi B, et al. High concordance of KRAS status between primary colorectal tumors and related metastatic sites: implications for clinical practice. Oncologist. 2008;13(12):1270-1275.

51. Chung KY, Shia J, Kemeny NE, et al. Cetuximab shows activity in colorectal cancer patients with tumors that do not express the epidermal growth factor receptor by immunohistochemistry. J Clin Oncol. 2005;23(9):1803-1810.

52. Hebbar M, Wacrenier A, Desauw C, et al. Lack of usefulness of epidermal growth factor receptor expression determination for cetuximab therapy in patients with colorectal cancer. Anticancer Drugs. 2006;17(7):855-857.

53. Saltz L. Epidermal growth factor receptor-negative colorectal cancer: is there truly such an entity? Clin Colorectal Cancer. 2005;5(Suppl 2): S98-S100.

54. Personeni N, Fieuws S, Piessevaux H, et al. Clinical usefulness of EGFR gene copy number as a predictive marker in colorectal cancer patients treated with cetuximab: a fluorescent in situ hybridization study. Clin Cancer Res. 2008;14(18):5869-5876.

55. Sartore-Bianchi A, Moroni M, Veronese S, et al. Epidermal growth factor receptor gene copy number and clinical outcome of metastatic colorectal cancer treated with panitumumab. J Clin Oncol. 2007;25:3238-3245.

56. Bengala C, Bettelli S, Bertolini F, et al. Epidermal growth factor receptor gene copy number, K-ras mutation and pathological response to preoperative cetuximab, 5-FU and radiation therapy in locally advanced rectal cancer. Ann Oncol. 2009;20(3):469-474.

57. Graziano F, Ruzzo A, Loupakis F, et al. Pharmacogenetic profiling for cetuximab plus irinotecan therapy in patients with refractory advanced colorectal cancer. J Clin Oncol. 2008;26(9):1427-1434.

58. Lurje G, Nagashima F, Zhang W, et al. Polymorphisms in cyclooxygenase-2 and epidermal growth factor receptor are associated with progression-free survival independent of K-ras in metastatic colorectal cancer patients treated with single-agent cetuximab. Clin Cancer Res. 2008;14(23):7884-7895.

59. Gonçalves A, Esteyries S, Taylor-Smedra B, et al. A polymorphism of EGFR extracellular domain is associated with progression free-survival in metastatic colorectal cancer patients receiving cetuximab-based treatment. BMC Cancer. 2008;8:169.

60. Garm Spindler KL, Pallisgaard N, Rasmussen AA, et al. The importance of KRAS mutations and EGF61A > G polymorphism to the effect of cetuximab and irinotecan in metastatic colorectal cancer. Ann Oncol. 2009; Jan 29. [Epub ahead of print]

61. Khambata-Ford S, Garrett CR, Meropol NJ, et al. Expression of epiregulin and amphiregulin and K-ras mutation status predict disease control in metastatic colorectal cancer patients treated with cetuximab. J Clin Oncol. 2007;25(22):3230-3237. 
62. Mutsaers AJ, Francia G, Man S, et al. Dose-dependent increases in circulating TGF-alpha and other EGFR ligands act as pharmacodynamic markers for optimal biological dosing of cetuximab and are tumor independent. Clin Cancer Res. 2009;15(7):2397-2405.

63. Tejpar S, Peeters M, Humblet Y, et al. Phase I/II study of cetuximab dose-escalation in patients with metastatic colorectal cancer (mCRC) with no or slight skin reactions on cetuximab standard dose treatment (EVEREST): Pharmacokinetic (PK), Pharmacodynamic (PD) and efficacy data. J Clin Oncol (Meeting Abstracts). 2007;25:4037.

64. Tejpar S, Peeters M, Humblet Y, et al. Relationship of efficacy with KRAS status (wild type versus mutant) in patients with irinotecan-refractory metastatic colorectal cancer (mCRC), treated with irinotecan ( $q 2 w)$ and escalating doses of cetuximab $(\mathrm{q} 1 \mathrm{w})$ : The EVEREST experience (preliminary data). J Clin Oncol (Meeting Abstracts). 2008;26:4001.

65. Davies H, Bignell GR, Cox C, et al. Mutations of the BRAF gene in human cancer. Nature. 2002;417(6892):949-954.

66. Samowitz WS, Sweeney C, Herrick J, et al. Poor survival associated with the BRAF V600E mutation in microsatellite-stable colon cancers. Cancer Res. 2005;65(14):6063-6069.

67. Ogino S, Nosho K, Kirkner GJ, et al. CpG island methylator phenotype, microsatellite instability, BRAF mutation and clinical outcome in colon cancer. Gut. 2009;58(1):90-96.

68. Di Nicolantonio F, Martini M, Molinari F, et al. Wild-type BRAF is required for response to panitumumab or cetuximab in metastatic colorectal cancer. J Clin Oncol. 2008;26:5705-5712.

69. Perrone F, Lampis A, Orsenigo M, et al. PI3KCA/PTEN deregulation contributes to impaired responses to cetuximab in metastatic colorectal cancer patients. Ann Oncol. 2009;20:84-90.

70. Jhawer M, Goel S, Wilson AJ, et al. PIK3CA mutation/PTEN expression status predicts response of colon cancer cells to the epidermal growth factor receptor inhibitor cetuximab. Cancer Res. 2008;68:1953-1961.

71. Sartore-Bianchi A, Martini M, Molinari F, et al. PIK3CA mutations in colorectal cancer are associated with clinical resistance to EGFRtargeted monoclonal antibodies. Cancer Res. 2009;69(5):1851-1857.

72. Prenen H, De Schutter J, Jacobs B, et al. PIK3CA mutations are not a major determinant of resistance to the epidermal growth factor receptor inhibitor cetuximab in metastatic colorectal cancer. Clin Cancer Res. 2009;15(9):3184-3188.

73. Zhou XP, Loukola A, Salovaara R, et al. PTEN mutational spectra, expression levels, and subcellular localization in microsatellite stable and unstable colorectal cancers. Am J Pathol. 2002;161(2):439-447.

74. Razis E, Briasoulis E, Vrettou E, et al. Potential value of PTEN in predicting cetuximab response in colorectal cancer: an exploratory study. BMC Cancer. 2008;8:234.

75. Frattini M, Saletti P, Romagnani E, et al. PTEN loss of expression predicts cetuximab efficacy in metastatic colorectal cancer patients. Br J Cancer. 2007;97:1139-1145.

76. Loupakis F, Pollina L, Stasi I, et al. PTEN expression and KRAS mutations on primary tumors and metastases in the prediction of benefit from cetuximab plus irinotecan for patients with metastatic colorectal cancer. J Clin Oncol. 2009;27(16):2622-2629.

77. Serova M, Ghoul A, Benhadji KA, et al. Effects of protein kinase C modulation by PEP005, a novel ingenol angelate, on mitogen-activated protein kinase and phosphatidylinositol 3-kinase signaling in cancer cells. Mol Cancer Ther. 2008;7:915-922.
78. Pencreach E, Guerin E, Nicolet C, et al. Marked activity of irinotecan and rapamycin combination toward colon cancer cells in vivo and in vitro is mediated through cooperative modulation of the mammalian target of rapamycin/hypoxia-inducible factor-1alpha axis. Clin Cancer Res. 2009;15:1297-1307.

79. Meier F, Guenova E, Clasen S, et al. Significant response after treatment with the mTOR inhibitor sirolimus in combination with carboplatin and paclitaxel in metastatic melanoma patients. J Am Acad Dermatol. 2009;60:863-868

80. Laghi L, Bianchi P, Orbetegli O, Gennari L, Roncalli M, Malesci A. Lack of mutation at codon 531 of SRC in advanced colorectal cancers from Italian patients. Br J Cancer. 2001;84(2):196-198.

81. Wheeler DL, Iida M, Kruser TJ, et al. Epidermal growth factor receptor cooperates with Src family kinases in acquired resistance to cetuximab. Cancer Biol Ther. 2009;8(8):696-703.

82. Boyd DD, Wang H, Avila H, et al. Combination of an SRC kinase inhibitor with a novel pharmacological antagonist of the urokinase receptor diminishes in vitro colon cancer invasiveness. Clin Cancer Res. 2004;10:1545-1555.

83. Huang F, Greer A, Hurlburt W, et al. The mechanisms of differential sensitivity to an insulin-like growth factor-1 receptor inhibitor (BMS536924) and rationale for combining with EGFR/HER2 inhibitors. Cancer Res. 2009;69(1):161-170.

84. Viloria-Petit A, Crombet T, Jothy S, et al. Acquired resistance to the antitumor effect of epidermal growth factor receptor-blocking antibodies in vivo: a role for altered tumor angiogenesis. Cancer Res. 2001;61:5090-5101.

85. Dempke WC, Heinemann V. Resistance to EGF-R (erbB-1) and VEGF-R modulating agents. Eur J Cancer. 2009;45:1117-1128.

86. Musolino A, Naldi N, Bortesi B, et al. Immunoglobulin G fragment $\mathrm{C}$ receptor polymorphisms and clinical efficacy of trastuzumab-based therapy in patients with HER-2/neu-positive metastatic breast cancer. J Clin Oncol. 2008;26(11):1789-1796.

87. Weng WK, Levy R. Two immunoglobulin $\mathrm{G}$ fragment $\mathrm{C}$ receptor polymorphisms independently predict response to rituximab in patients with follicular lymphoma. J Clin Oncol. 2003;21(21):3940-3947.

88. Zhang W, Gordon M, Schultheis AM, et al. FCGR2A and FCGR3A polymorphisms associated with clinical outcome of epidermal growth factor receptor expressing metastatic colorectal cancer patients treated with single-agent cetuximab. J Clin Oncol. 2007;25(24): 3712-3718

89. Bibeau F, Lopez-Crapez E, Di Fiore F, et al. Impact of Fc $\{$ gamma $\}$ RIIa-Fc $\{$ gamma $\}$ RIIIa polymorphisms and KRAS mutations on the clinical outcome of patients with metastatic colorectal cancer treated with cetuximab plus irinotecan. J Clin Oncol. 2009;27(7):1122-1129.

90. Levy EM, Bianchini M, Von Euw EM, et al. Human leukocyte antigen-E protein is overexpressed in primary human colorectal cancer. Int J Oncol. 2008;32(3):633-641.

91. Levy EM, Sycz G, Arriaga JM, et al. Cetuximab-mediated cellular cytotoxicity is inhibited by HLA-E membrane expression in colon cancer cells. Innate Immun. 2009;15(2):91-100.
Cancer Management and Research

\section{Publish your work in this journal}

Cancer Management and Research is an international, peer-reviewed open access journal focusing on cancer research and the optimal use of preventative and integrated treatment interventions to achieve improved outcomes, enhanced survival and quality of life for the cancer patient. The journal welcomes original research, clinical \& epidemiological

\section{Dovepress}

studies, reviews \& evaluations, guidelines, expert opinion \& commentary, case reports \& extended reports. The manuscript management system is completely online and includes a very quick and fair peerreview system, which is all easy to use. Visit http://www.dovepress.com/ testimonials.php to read real quotes from published authors. 